\title{
Grecità (e romanticismo) \\ nella "mitologia moderna" \\ di Alberto Savinio
}

Ignorare lo stato di magia che ci ha preceduti significa d'altra parte non sentire lo stato di magia in mezzo al quale viviamo. Come adulti che hanno dimenticato la propria infanzia...

"Lasciate che l'infanzia" (Opere 730).

La forza immaginativa di Alberto Savinio trae origine all'interno del patrimonio mitologico classico che fornisce presupposti e strumenti grazie ai quali creare una narrazione che esce dalla dimensione del quotidiano e si costruisce in un gioco di memoria e invenzione. Tuttavia, si deve registrare un'attenzione non esaustiva né definitiva, da parte della critica, alla riabilitazione mitologica attuata dall'autore (soprattutto in riferimento al significato che assume il mito dell'ermafrodito), senza che sia mai stata approfondita e risolta in maniera organica la dinamica psicologica che connette la centralità del recupero mitologico alla dimensione biografica, per così dire infantile, dello scrittore. Sembra opportuno operare qui uno spostamento di prospettiva dal molto discusso rapporto letterario dell'autore con le varie avanguardie novecentesche alla questione della "classicità" di e in Savinio. L'interesse al rifacimento della mitologia consente meglio di chiarire in che termini Savinio fosse interessato alla produzione di un linguaggio assolto dai doveri della rappresentazione naturalistica, ma in una sempre maggiore attenzione al bagaglio culturale antico, inteso come riscatto e studio diretto dei testi di origine, dopo l'assoluto rifiuto futurista in Italia (e prima ancora romantico in Europa) delle tematiche mitologiche. L'adozione della prospettiva classica ha riflessi fondamentali nella soluzione tematica in sede narrativa, teatrale e artistica; e anche in ambito più specificamente linguistico l'autore non prescinde dal rivendicare il proprio diritto ad esercitare la professione di erudito nello scioglimento poetico di etimologie antiche. ${ }^{1}$ Il repertorio classico saviniano non viene ad assumere i connotati di una poetica formale del culto della parola nobile, ricercata, antica, quanto testimonia dell'estrema significatività assunta 
dalla propria "grecita", sentita come "sentimento dell'infanzia", da cui importa non allontanarsi mai. ${ }^{2}$ Lo scrittore nasce e si forma ad Atene, "ad Atene ci è nato... Atene la conosce bene... Atene la ricorda bene" (afferma Savinio in una usuale terza persona grammaticale), tanto da fare di quel repertorio di immagini antiche il suo sistema narrativo (Opere 69). Savinio gioca costantemente ad amalgamare il repertorio classico che ruota intorno ad Atene alla propria "grecità" ciò̀ alla memoria individuale di quei luoghi. La necessità appare qui di indirizzarsi decisamente al rapporto fra l'analisi testuale di certi motivi "greci" (l'ermafrodito, la metamorfosi) in quanto di memoria classica e autobiografica e la funzione attribuita all'arte nella costruzione di un proprio universo in cui gli echi della cultura classica si assemblano insieme in una scrittura di straordinaria qualità inventiva e parodica.

Le quattro sezioni di quest'articolo tendono a individuare certe costanti dalla prima produzione letteraria: il fantastico che si costruisce sulla ripresa "moderna" della mitologia greca, in opposizione al fantastico onirico surrealista (primo paragrafo); l'ambiguità e doppia articolazione, moderna e classica, della poetica saviniana, che ha la sua prima formulazione nella metafora dell'ermafrodito nel primo romanzo, intitolato Hermaphrodito (secondo paragrafo) e che a me appare connessa al bisogno di uno sguardo non naturalistico, estremo, capace di irrompere in un mondo inedito. Questo e testimoniato da molta della successiva narrativa saviniana; qui si rivolgera attenzione al romanzo Casa "La vita" (terzo paragrafo). L'importanza di tale passaggio è confermata dall'ermafrodito come simbolo di un di più a cui guardare e un di più da rappresentare. Tale figura mitica viene percepita da uno sguardo capace di uscire dalla categoria temporale del presente con una costante disposizione all'immaginazione, all'avventura ripetitiva del meraviglioso mitologico. L'attrazione per nuovi e diversi punti di osservazione implica un recupero delle categorie della grecità (l'occhio come memoria dell'infanzia) e della classicità (la sostanza mitologica della storia di Orfeo) e approda in una realtà dove si assiste alla metamorfosi delle essenze. Savinio, innestato di Ovidio, si appropria del concetto di trasmutabilità della realtà, secondo le moltiplicazioni in nuove entità raccontate nelle Metamorfosi.

Inoltre, nel panorama novecentesco della critica, mancano voci dedicate a quello che sarebbe poi divenuto il leitmotiv fantastico della produzione del Nostro in relazione al recupero e all'individuazione delle suggestioni della letteratura fantastica dell" 800 francese (Prosper Merimé, Gerard de Nerval, Teophile Gautier, Lautréamont). ${ }^{3}$ Mi propongo, nell'ultima sezione di questo studio, di ricostruire una fonte nell'ambito di un episodio contenuto in Casa "La vita", in relazione alla ricorrenza nell'arte savininana del tema della morte-metamorfosi, ospitato come dimensione mitologica del passato e del presente e quindi immagine del perenne movimento, della poliedricità della realtà. Quindi, si procede a una lettura testuale di due racconti di Casa " $L a$ vita" che consentono, l'ultimo, "Flora", di soffermarsi sulle fonti presenti alla 
narrazione saviniana; l'altro, "Il Signor Münster", di coordinare la morte a fenomeno simile a quello della metamorfosi, nella (ri)nascita al sentimento del passato antico, sulla lezione del pensiero di Nietzsche.

\section{Il fantastico mitologico}

Nel 1940 André Breton curava la prima edizione della celebre Anthologie de l' humour noir (da cui prendo la citazione del mio titolo) e vi includeva Savinio come precursore della poetica surrealista. Breton riconosceva Alberto Savinio e il fratello Giorgio De Chirico come anticipatori "du mythe moderne encore en formation [...] de l'interrogation métaphysique propre à cette epoque" (231), stabilendo il 1914 come limite cronologico. Esiste una, sia pur tarda, risposta di Savinio alla questione affrontata da Breton, in cui si esprimono dubbi riguardo alla relazione tra le proprie opere ed il surrealismo, con presa di distanza dalla libertà e casualità inventiva di quell'avanguardia. Nella prefazione al romanzo Tutta la vita, lo scrittore, come è noto, precisa la sua esigenza di ordine e controllo sulla materia:

il surrealismo [...] è la rappresentazione dell'informe ossia di quello che ancora non ha preso forma [...]. Quanto a un surrealismo mio, se di surrealismo si può parlare, esso è esattamente il contrario [...] non si contenta di rappresentare l'informe e di esprimere l'incosciente, ma vuole dare forma all'informe e coscienza all'incosciente [...]. Nel surrealismo mio si cela la volontà formativa e, perché non dirlo? una specie di apostolico fine. (7-8)

Sulla scia di Breton, altri lettori hanno scambiato il linguaggio di Savinio con quello del sogno, inglobandolo, entro certi limiti, nell'immaginario verbale e pittorico del surrealismo. Piuttosto pare che il fantastico in Savinio assuma una dimensione ludica sì, ma premeditata e costruita, che non prende le mosse dalla trascrizione fluttuante e casuale dell'esperienza automatica, realizzata dal surrealismo per così dire "siglato". ${ }^{4}$ Di teorie psicanalitiche, come ben si sa, i surrealisti si servirono per esplorare il mondo del sogno e dell'inconscio, interessandosi a stati quali l'automatismo e la follia. Sembra, al contrario, che gli esperimenti di Savinio non siano rivolti soltanto a comunicare la forma alternativa del reale, affidandoci agli spazi sconosciuti della "surrealtà", ma figurino alla costruzione di una dimensione passata, mitologica, ricuperabile soltanto tramite l'ironia. Fra le numerose testimonianze è rilevante citare quella contenuta in "Uscita dal labirinto": "Compito del poeta è di trovare l'uscita del labirinto. Questo il grande compito del poeta. Trovare l'uscita e indicarla agli altri, ai compagni uomini, ai fratelli uomini. Si dice tanto che poeta viene da poieo e che poieo significa fare. Non fermiamoci qui. Fare, significa trovare l'uscita dal labirinto" (Opere 911). Savinio tenta di ri- 
ordinare il caos in una struttura non ortodossa, aperta, onnicomprensiva. Nulla diventa allora di più estraneo all'arte quanto i meccanismi dell'inconscio, data la forza costruttiva che si attribuisce a quella. Il fantastico, quindi, non si alimenta dal sogno, anche se di teorie oniriche l'autore si interessa leggendo Freud, ${ }^{5}$ ma viene riscattato da una scrittura formativa e cosciente delle proprie scelte linguistiche e tematiche: nel tentativo perenne di cogliere in termini ironici l'affiorare, in forma di immagini mitologiche, della sostanza latente dell'esistenza umana.

Non necessita insistere qui sull'influenza dei movimenti avanguardistici in Savinio, ${ }^{6}$ soprattutto sul tanto studiato plurilinguismo della prima produzione, notato immediatamente da Papini nella recensione a Hermaphrodito nel 1919 in "Il Resto del Carlino"; " quanto leggere, a livello di organizzazione tematica, l'incontro fra presente e passato nella creazione di un fantastico tutto a sé, che risulta per il lettore imprendibile, esorcizzante, demistificante. L'eccezionale unità tematica verrà considerata qui a partire dalla prima prova letteraria, e quindi dalla prima riscrittura mitologica, contenuta in Hermaphrodito del 1918.

Già dalla sezione finale di Hermaphrodito, la più articolata e narrativa, intitolata "La partenza degli argonauti", Savinio presenta situazioni al limite della realtà tramite l'adozione moderna della mitologia. L'erudizione e la conoscenza diretta dei testi greci (ampiamente testimoniata dagli scritti di carattere giornalistico) gli consente di rileggere in termini paradossali e grotteschi le favole passate: viene prestata particolare attenzione al mito di Amore e Psiche (nei romanzi La nostra anima, Angelica o la notte di maggio), al viaggio omerico e agli eroi greci (nei testi Capitan Ulisse, Achille innamorato, in "Walde Mare" in Casa "La vita" e in "La partenza dell'argonauta" in Hermaphrodito), alla morte eroica (in Alcesti di Samuele; in "Il Signor Münster" in Casa "La vita"), alla metamorfosi (in "Flora" e "Mia madre non mi capisce" in Casa "La vita"). L'atteggiamento verso l'apparato mitologico denota da una parte una forte attrazione verso l'ordine simbolico che i racconti mitici tramandano, dall'altra un moderno distacco che permette letture grottesche, ridicole e comiche delle favole antiche. Il capovolgimento di prospettiva non annulla, ma è generato dalla grande attrazione per quelle fantasie classiche. Come sostiene Jesi, anche a qualsiasi resa comica di passati apparati culturali sopravvive un ambiguo amore per quegli stessi: quello che connota la parodia è la diversa maniera di leggere certe immagini, non riconoscendo più nella tradizione l'unico percorso attuabile. ${ }^{8}$ Le testimonianze sono numerose; soltanto per ampliare il raggio di lettura in ambito comparativo, si ricordi la riflessione di Thomas Mann a proposito del suo 'idillio' Herr und Hund, che rivede l'idillio germanico in luce ridicola: "[...] insomma, la mancanza di vera e propria ingenuità si manifesta nella tendenza parodistica, e così, da questo piccolo evento poetico si dovrebbe almeno dedurre la legge o la definizione che l'amore a uno spirito artistico che non si crede ormai più possibile 
produce la parodia" (Jesi 190). La stessa opera di Ezra Pound è contrassegnata dalla presenza ininterrotta di immagini mitiche, in un gioco ambiguo fra parodia e devozione nei confronti di quelle stesse rappresentazioni, come testimonia la celebre epifania dionisiaca del Canto II dei Cantos. Anche Bertold Brecht intuisce la necessità di uscire da un rapporto schiacciante e devozionale con i grandi autori del passato, a causa del terrorismo esercitato sui moderni dai classici. Ma contemporaneamente comprende che: "certi testi del passato non parlano più per colpa nostra che per colpa loro, e la modemità non è soltanto in ciò che è esterno ma è soprattutto in cid che è interno, se adeguatamente interrogato [...]. Solo un'interpretazione storica, cioè in qualche modo filologica, che entri dentro un testo di quella natura può forse dare a quel testo la sua modernita" (Raimondi 89).

Una lettura simile della modemità elabora Nietzsche nella seconda delle quattro "Considerazioni inattuali", pubblicate fra il 1873 e 1876, dal titolo Utilitd e svantaggi della storia per la vita. La polemica del filosofo si dirige contro il pensiero borghese del secolo, nel programma di liberazione dallo storicismo inteso come ossequio passivo "gesto meccanico di assenso a ogni potenza storica". L'aggettivo moderno viene ad assumere rilevanza proprio in riferimento a coloro che si soffermano sul passato: "Poiché noi siamo il frutto delle generazioni precedenti, siamo anche il frutto delle loro passioni, dei loro sviamenti, dei loro errori e persino dei loro delitti. Non ci si pud liberare da tutta questa cultura...noi deriviamo da quelli" (33). La conoscenza della cultura che ci ha preceduti deve essere gestita e coordinata alla fisionomia del presente, talché un eccesso di storia diventa dannoso all'esistenza, se non intelligentemente "animato dalla fresca vita del presente". Il recupero, dunque, deve essere abilmente bilanciato e costruttivo:

La conoscenza del passato in tutti i tempi non è desiderabile che quando è al servizio del futuro e del presente, non quando serve a indebolire il presente, non quando sradica le energie del futuro [...]. Noi moderni non abbiamo nulla di nostro; soltanto caricandoci e sovraccaricandoci di epoche, di costumi, di arti, di filosofia, di religioni e conoscenze straniere, diventiamo degni di rispetto; enciclopedie ambulanti [...]; cosicché la cultura moderna è essenzialmente interiore: sulla copertina il legatore ha stampato un titolo di questo genere "Manuale della cultura interiore per barbari esteriori". (34-38)

Savinio conosce l'opera di Nietzsche (come è stato recentemente dimostrato dalla Tordi) tanto che si coglie una certa vicinanza fra la tesi che informa il pensiero del filosofo e l'interpretazione saviniana della morte nel racconto "Il Signor Münster": una (ri)nascita al passato in cui le categorie di presente, passato, futuro si amalgamano insieme. Lo vedremo in breve. In termini più ampi la frequentazione della filosofia nietzschiana permette di individuare la fisionomia della classicitd come grecitd in Savinio, volta ad una riappropria- 
zione giusta di quei parametri immaginativi, ma, nello stesso tempo, ad una ricezione della novità romantica. Il libero gioco della vena poetica se qualifica e fa rivivere il proprio passato, tiene anche doverosamente presente 1"“evoluzione" e 1" "interno" della realtà: classicità, dunque, come dimensione dell'infanzia; romanticismo come garanzia di modernità e movimento. L'affermazione di siffatta poetica che definirei ossimorica è ben formulata in "Lasciate che l'infanzia".

L'Italiano pensa gli uomini e le cose già formati. Una volta per sempre. In maniera definitiva. Non pensa alla formazione delle cose. Non pensa come le cose si siano formate né perché. Classicismo assoluto. Classicismo naturale. Classicismo fin dalla nascita. Si parte dal classicismo [...]. Non solo si oppone una "sana" ostilità alla evoluzione delle cose. Meglio ancora si oppone una sana indifferenza. Che di più classico di questa indifferenza? Evoluzione è moto e mutamento: concetti romantici [...] scoprire come le cose nascono [...] arrivare all' "interno" delle cose [...]. D'altra parte uomini popoli che hanno rotto i ponti tra sé e il proprio passato: uomini che hanno rotto i ponti tra sé e la propria infanzia. Adulti assoluti. Che grandi mutilati!. (Opere 730)

Da qui si giunge all'uso dell'ironia, come atteggiamento di partecipazione e distacco nei confronti della propria materia. Soltanto recuperando l'etimologia greca di eironeia, in quanto linguaggio di finzione, abbassamento, dissimulazione, è possibile conservare la serietà del passato e perseguire l'"apostolico fine" dell'arte:

Solo l'ironia ci dirà vivo il passato. Solo ci ridà la serietà del passato-di quello che per noi non ha più serietà, perché non partecipa più della nostra vita [...]. Solo l'ironia ci unisce a quello che per noi vivamente non è più [...]. Si vuol togliere davvero dalla noia le riesumazioni classiche? Siano intinti nell'ironia [...]. L'ironia non è ironica. L'ironia è seria. Profondamente seria. E pia. (Opere 765)

Savinio nel dialogo con le storie antiche tende a cogliere una costante perenne dell'esistenza umana (anche per motivi biografici), ma nello stesso tempo colma la distanza mentale e temporale che separa i moderni da quei racconti greci con una trasfigurazione del mito in epifania moderna. Il tentativo di recuperare un'impossibilità rivela la nostalgia verso quel tempo che concepiva il mito come idea, nell'incapacità di spiegare in diverso modo ogni scienza umana. Ho già indicato in altra sede il nesso teorico fra la concezione saviniana e quella vichiana del mito: nel riconoscimento, in entrambi, della prima manifestazione del linguaggio poetico nelle favole e nell'esplicazione dello stretto legame fra conoscenza mitica e origine del linguaggio della poesia. Come Vico nel Libro II della Scienza Nuova ("Della logica poetica") individuava la genesi del mito nel rapporto con la letteratura, così Savinio asserisce 
nel 1943 l'equivalenza fra leggenda e parola nelle pagine del romanzo Casa "La vita":

Nella lingua di Omero mito significa semplicemente parola. Gli eroi di Omero parlano come voi e me, solo che usano miti come noi usiamo parole. Si restituisca la parola omerica al suo valore, e il mitismo che rimarrà sarà il solo, il solito mitismo che è nelle parole dei poeti, nelle nostre. (273)

Le immagini mitologiche diventano, secondo una lettura moderna del mito, fenomeni, idee. Non il mito come creazione emblematica della conoscenza, né come forma narrativa di elaborazioni filosofiche e metafisiche, ma il mito coincidente col momento cognitivo umano, l'unica struttura linguistica e poetica. L'interpretazione e la ricerca del significato originale di tali iconografie, cui alcuni critici aspirano, diventa quindi impossibile. Infatti l'appropriazione moderna del mito traduce in Savinio una nostalgia per quell'età in cui le forme mitiche assumevano connotazione universale. Pertinente qui si presenta la prospettiva di Kermode, nella distinzione tutta moderna fra miti e fiction:

We have to distinguish between myths and fictions. Fictions can degenerate in myth whenever they are not consciously held to be fictive [...]. Myth operates within the diagram of ritual, which presupposes total and adequate explanations of things as they are and were; it is a sequence of radically unchangeable gesture. Fictions are for finding things outh and they change as the needs of sense-making change. Myths are the agent of stability, fictions the agents of change. Myths call for absolute, fictions for conditional assent. Myths make sense in terms of a lost order of time, illud tempus as Eliade calls it; fictions, if successful, make sense of the here and now, hoc tempus. (38)

Savinio, come Vico, supera l'incrostazione razionale-illuministica operata sul mito dall'uomo moderno. Pur accogliendo la narrazione favolistica in modo ironico, vi ravviva l'atmosfera di mistero, il suo senso di incomprensibilità e mobilità. Dell'ironia si serve non come gioco sull'essenza del mito puro in senso vichiano, in quanto simbolo significante, ma per stabilire il mito come prodotto ibrido, quasi una sostanza teatrale, grottesca e assurda. La contraddizione fra recupero vichiano e lettura ironica testimonia anche della complessità e imprendibilità del pensiero di Savinio che rifugge sia da una sistemazione critica tout court che da programmi teorici definiti una volta per tutte. La multiformità è significativamente eletta da Savinio centro della propria esistenza poetica, nel rifiuto della coerenza dogmatica:

Molto ammirato è l'uomo che rimane fermo sulle stesse opinioni e si dice di lui che è un 'carattere'. A me questa specie di uomo non va. Sarà un 'carattere', ma è anche una 
testa di legno. A me piace l'uomo che nega ciò che ieri affermava. È segno di curiosità, di ricerca, di vedere molto e lontano; di sapere che la verità è multipla. (Alcesti 15)

\section{Ermafrodito in Hermaphrodito (e oltre)}

Sembra, a questo punto, necessario tener presente la prima immagine classica recuperata dall'autore, quella dell'androgino, in rapporto alle diverse gradazioni che assume poi nell'opera letteraria saviniana come metafora privilegiata per rappresentare gli opposti. Mi riferisco al romanzo d'esordio di Alberto Savinio, Hermaphrodito. Se di romanzo si pud parlare, per la struttura fortemente smembrata, sbilanciata e enigmatica che stride al confronto con l'impianto narrativo tradizionale. La fortuna critica di questo testo viene segnata da un lato dall'impossibilità di collocare quelle pagine nel panorama novecentesco, dall'altra da un certo disorientamento che suscito nei lettori, anche quelli di professione: al punto che si rivelò molto più facile per Papini, nella sua recensione del 1919 , indicare per lo più quello che il testo non era, piuttosto che definirne i nuovi tratti. ${ }^{9}$ Della ricostruzione filologica del romanzo, mi sono occupata in altra sede: qui importa soltanto ricordare la pubblicazione per la prima volta in volume a Firenze nel dicembre 1918 nelle edizioni della "Libreria della Voce" e insistere sulla soluzione antiromanzesca che connota, in realtà, tutti i testi saviniani: degli antiracconti che si costruiscono sotto le costanti di "fluidificazione", dissacrazione, digressione (Cirillo 100). L'analisi interpretativa del motivo mitologico dell'androgino comporta attenzione al titolo stesso, Hermaphrodito, che ripristina linguisticamente la lezione più arcaica e suscita non poche perplessità, dato che il romanzo non recupera la storia classica dell'Ermafrodito. ${ }^{10} \mathrm{La}$ critica si è ampiamente posta interrogativi inerenti all'elaborazione della funzione ermafroditica che in quelle pagine confluisce. La discussione intorno alla lettura del titolo si e orientata (e risolta) a sottolineare la situazione di "oscillante equivocità" (Bramanti 19) tipica della coniunctio oppositorum, che definisce quel testo e quelli successivi: una tendenza a guardare e privilegiare gli opposti contemporaneamente, una condizione esistenziale che permette di tramutare in metafora, naturalmente carica di significazioni ben connotate, l'ideologia saviniana, una cifra, un simbolo di un sistema letterario e artistico ambivalente, poliversatile, ermafroditico, appunto. Nel primo romanzo, il motivo dell'ermafrodito compare esplicitamente soltanto in alcuni capitoli: in "La partenza dell'argonauta" ("Sono l'opposto di Maurizio Maeterlink - voglio dire non ho l'animo bacato da nessun ermafroditismo idealistico - epperciò il divagare ad occhi aperti mi ripugna") in cui si dichiara la propria distanza da un certo tipo di ermafroditismo negativo, idealistico, onirico, da sognatore ad occhi aperti; in "Il Religioso" ("Ivi m'intoppai in un ebreo [...]. Prognosticai in lui un misto dei due sessi [...]. Lo indovinai malato di un doppio motore genitale e 
sofferente così degli ovari che dei testicoli") dove si ricompone il conflitto dei contrari nella comparsa di un Ermafrodito come simbolo unificatore. Infatti si continua: "incinto tutti i nove giorni dell'anno e mammelluto come un Tiresia, l'uomo sul tetto butta da meato puzzolente grappoli di carne per terra che si ritorcono, poi si levano e camminano, indi crescon con vigore - insufflati da un calore dilatante - e finalmente si fan uomini seriosi e dottrinari di morale". E, infine, il tema ritorna nel breve capitolo "Il Lirico" ("Per quanto camminassi, non riuscii a slacciarmi dalla voce dello strano ermafrodito, che tagliava l'epoche da parte a parte"). Ma lo stesso motivo ermafroditico compare costantemente in molti altri testi successivi: si vedano in particolare Tragedia dell' infanzia (Apollo/Apolla, il misterioso essere oggetto di curiosità da parte del piccolo Andrea), La casa ispirata (la funzione ermafroditica rimanda ad un "ricordo losco e tenebroso"), Capitan Ulisse (l'integrazione fra i due sessi è necessaria per essere attori), Infanzia di Nivasio Dolcemare (ermafrodito è ideale di perfezione), Ascolto il tuo cuore, cittd (il connotato primario negli "uomini della Poesia, in questi uomini che sono assieme donne"), in Tutta la vita ("Le due sorelle morte rivissero effettivamente nel fratello vivo. Igeo a poco a poco divento uomo e assieme donna, e anche nella mollezza dei tratti, nella rotondità dei fianchi, nella voce, nei gesti, nei gusti [...]"). Tutto ciò costituisce un esempio paradigmatico della ricorrente esigenza di osservare e rappresentare gli opposti nella narrativa di Savinio (la dicotomia passato/ presente; moderno/antico; romantico/classico), nel proposito fondamentale di rappresentare la poliedricita dei fenomeni. Inoltre, le osservazioni sin qui svolte permettono, con diversi gradi di coerenza, di collegare il tema della praesentia con quello della osbervatio oppositorum e di indicare, nel contempo, la base mitologica della tematica della curiositd visiva in Savinio. Vale la pena, prima di soffermarsi sulla ricezione delle principali fonti classiche, accennare brevemente che anche nella pittura dell'avanguardia primo novecentesca il referto iconografico è costante: Ermafrodito appare per lo più sdraiato e nudo in linea con la tipologia largamente presente nella scultura ellenistica. Fra le varie raffigurazioni che ho già notato in altra sede, occorre qui ricordare di Savinio l'olio su tela del 1927 "Le sommeil de l'Hermaphrodite" "e una tempera ad olio su compensato dal titolo "Riposo di Hermaphrodito", ${ }^{12}$ risalente, forse, all'epoca della seconda ristampa del romanzo omonimo. Il dipinto è un'esaltazione della cosmogonia divina: l'occhio di un demone vola in cielo, mentre ermafrodito giace addormentato di schiena. II quadro nasconde il volto e la compresenza dei due sessi nell'androgino, la cui testa è sostituita da una spirale con lunghi capelli che si adagiano fino alla vita: ma Ermafrodito non è messo in condizione di guardare, in quanto la spirale del capo è reclinata nel ripiego delle braccia. È fondamentale qui l'accostamento fra la tematica visiva e la simbologia mitica del doppio nell'androgino che non è soggetto guardante, ma oggetto osservato. La tesi 
condotta fin qui sembra testimoniata nell'analogia fra iconografia pittorica e immaginativa letteraria in quanto l'artista stabilisce un nesso preciso (e a mio avviso indissolubile) fra la figura dell'ermafrodito come metafora della complessità del reale e la comparsa di uno sguardo che coglie e svela quella complessità. Darò nel prossimo paragrafo testimonianza narrativa della raffigurazione di una vista privilegiata in due racconti di Casa "La vita". Lo sguardo del quadro ("Riposo di Hermaphrodito") tuttavia, non appare stabile, ma vola in cielo, imprendibile e sempre in movimento, per alternare i suoi punti di vista. A siffatta vista e permesso di intendere le alterazioni cui va soggetta la realtà, fino alla chiarificazione del processo fantastico della metamorfosi. Anche a questa proposta offriro fra breve il necessario apporto testuale.

L'ermafrodito nelle fonti classiche diventa simbolo o della perfezione in quanto completezza (nel Simposio di Platone) o al contrario della separazione (nel Convito di Platone, 14, 309-10), e del dolore. ${ }^{13}$ In Nuova Enciclopedia il rimando al Convito è chiaro nella decisione degli Dei di limitare il potere degli androgini: "i coniugi ci apparirebbero quali veramente sono, mostri forniti di quattro gambe e di quattro braccia, con due nasi e due bocche e che camminano come ragni enormi. Chi è l'uomo di noi due e chi è la donna? Giove tagliò nel mezzo gli uomini-donne perché troppo pericolosi alla sua sovranita" (341). Anche la diretta conoscenza delle Metamorfosi di Ovidio permette a Savinio di collegare la creazione mitopoietica al tema del dolore, della scissione e perdita. Moroni ricostruisce l'immagine dell'ermafrodito nell'immaginario della metafisica del Primo Novecento; a suo parere (e giustamente) tale immagine in Savinio potrebbe essere vista come omologa alla condizione dell'artista in una fase definibile come "modernista", in cui emergono risposte caratterizzate da una complessa dinamica:

In Savinio's writings, the memory and the heritage of the myth of the hermaphrodite play a basic role in a complex linguistic and cultural preposition. Savinio's autobiographical "I" can be seen to react to the flattening out of values in the modern world by constructing his own mode of hermaphroditic presence. The narrative space functions as a space of linguistic otherness, in which the characteristics of the hermaphrodite and of a modernist version of it - offer a sphere of transgressive negativity, and, at the same time, a sense of lost fullness. ${ }^{14}$

La metafora dell'ermafrodito ricompare, quindi, a riprova della nostalgia di Savinio per quella completezza mitologica, e, a sua volta, motiva l'atteggiamento ironico-parodico per un'impossibilità perduta. Un'operazione che proietta ironicamente i propri referenti, secondo la posizione auto-assunta di "ipocrita", dichiarata nelle pagine del primo romanzo:

Chiunque mi chiami ipocrita non mi offende. Sciogliamo il sostantivo: ipocrita indica colui che esamina da sotto - una posizione che contiene il corollario di due altre 
posizioni precedenti: l'esame superno e quello interno. Intendere tutto, penetrando nel tutto. (207)

\section{Lo sguardo ermafroditico}

Sembra lecito essere partiti dalla metafora di Ermafrodito non solo perché è la prima immagine mitologica rielaborata, ma anche perché l'autore, quasi quarant'anni più tardi, e dopo una produzione letteraria che rientra in una struttura più composita e narrativa, insegna la continuità delle sue opere, proprio da Hermaphrodito: laddove il primo romanzo non viene mai negato nella sua sperimentazione, ma, al contrario, dichiarato padre dell'intera produzione. E qui Savinio non si riferisce soltanto a quella letteraria. Ermafrodito diventa generatore, procreatore, e simbolo di ciò che seguirà: un Tiresia, che contiene in sé entrambi i sessi, e possiede capacità auto-riproduttive. Siamo alla vigilia della seconda ristampa del romanzo (per Garzanti nel 1947), Savinio compone una fondamentale guida alla lettura della propria opera, scegliendo di (ri)proporre soltanto alcuni dei capitoli contenuti nell'edizione del romanzo del 1918, introducendone, fra l'altro, alcune varianti. Leggiamo da "Piccola Guida alla mia Opera Prima", adesso ripubblicata nell'ultima edizione di Hermaphrodito:

Io dopo trent'anni e più di cammino letterario, mi volto e vedo un'ombra che si parte da me come la coda del pavone e ripete in sagoma allungatissima le mie gambe, le mie spalle, la mia testa a palla [...]. Per ventinove anni non riaprii Hermaphrodito [...]. Sono io il padre di Hermaphrodito o il figlio? Il padre secondo il piu apparente cammino del tempo, che pone in fila i figli dopo i padri e i padri prima dei figli; il figlio secondo un cammino meno apparente del tempo, e, con parole d'oggi, secondo il tempo relativo, che consente ai padri di nascere dai propri figli. Ho guardato Hermaphrodito in faccia e senza paura. Mi sono riconosciuto intero nella sua faccia. Tutto che io sono nasce da lì. Tutto che io ho fatto viene da lì. Non c'è idea, non c'è pensiero, non c'è concetto, non c'è sentimento, non c'è immagine [...] che non tragg a da quella "pustola" e da quel "bubbone", indecente l'una e malefico l'altro, ma straordinariamente fecondi ambedue .

A questo punto diventa ancora più lecito insistere sulla struttura di quel romanzo, per il duplice fine di ricondurre alla significazione ermafroditica del doppio l'attenzione saviniana allo sguardo estremo; e per individuare proprio in quella visione surreale l'elemento costruttivo e centrale dell'altro romanzo di cui qui ci si occupa, Casa "La vita". Si potrà giungere così all'ultima tappa di questa ricerca che propone il tema della metamorfosi come riesumazione mitologica e modello gnoseologico di un al di là della realtà. Un percorso consequenziale che richiede la spiegazione di ogni singolo passaggio. 
La prima parte del romanzo Hermaphrodito contiene il sottotitolo "Microscopio-Telescopio" due maniere per alterare l'immagine, che indicano attrazione e curiosità per il potere dello sguardo come deformazione visiva, per il rovesciamento del reale che emerge dalla letteratura fantastica del Primo Novecento. L'inquietudine e insicurezza si affermano largamente fra gli scrittori di quel periodo in quanto strettamente connesse al nuovo modo di vedere, alla nuova abilità di interpretare questioni moderne: a ritroso finiamo per approdare al cannocchiale di Hoffmann, che nella capacità di duplicazione o comunque deformazione, causava nel racconto Der Sond mann (1816) l'elemento angoscioso, das Unheimlich (il "perturbante", così come Freud lo intese).

"Microscopio-Telescopio", dicevo: due strumenti ottici che presentano caratteristiche diametralmente opposte, ma che nello stesso tempo fanno fare prodigi alla vista umana: l'uno permette l'osservazione di oggetti di dimensioni inferiori alla capacità dell'occhio umano; l'altro osserva da lontano, ingigantendo i contorni posti a grande distanza. L'interesse per le tecniche in grado di deformare la vista naturale, Savinio lo rivela già dal testo del 1914 "Chants de la mi-mort" in cui compare una sezione sottotitolata "Episcope", apparecchio usato per le proiezioni di immagini riflesse tramite uno specchio. La dicotomia lontano-vicino segna il rifiuto della rappresentazione naturalista e insieme attribuisce alla scrittura una funzione e un dovere di spingersi oltre. Le tracce della dimensione visiva nella narrazione saviniana sono testimoniate anche dai molteplici interventi di Savinio sul potere dello sguardo; fino alla riflessione sulla costante raffigurazione dell'occhio nelle sue pitture. Nell'articolo "La Messa solenne" il motivo si illumina di una sua dimensione biografica e culturale: e ciò è fondamentale ir: quanto vi confluiscono indizi per la definizione della poetica della "grecita" saviniana come patrimonio di immagini strettamente coordinato alla categoria dell'infanzia:

L'infanzia io l'ho consumata in Grecia e nelle mie passeggiate obbligate io insistevo a entrare nelle chiese greche [...]. E poiché [...] l'Occhio di Dio è sempre presente nella rudimentale decorazione delle pareti, chiuso dentro un triangolo che nel giallo del suo colore esprime l'idea dell'oro, circondato da ciglia nere e simili alle zampe di alcun oggetto mostruoso, e con la pupilla enorme, nera, priva di riflesso, e fissa in mezzo al bianco della sclerotica, l'idea di quel divino e perseguitante strumento ottico aveva acquistato per me una sua realtà fisica. Quali effetti ha avuto nella mia vita psichica $e$ mentale l'infantile ossessione dell'occhio di Dio? Effetti profondissimi certo e indelebili se in tante mie immagini letterarie, come dimostra un esame della mia opera, e soprattutto in tante mie pitture, il tema dell'Occhio ritorna insistente: l'Occhio solitario in mezzo alla fronte, l'Occhio Eterno, l'Occhio cui nessuno sfugge né nulla. (Scatola Sonora 73 )

L'articolo "Anadioménon" contribuisce a precisare l'impossibile trasparenza del reale e la conseguente deformazione visiva; addirittura in "Mistero dello 
sguardo" si amplia questa prospettiva nell'elogio dello strabismo come alterazione che presuppone il fascino dello sguardo doppio, ermafroditico. È la memoria della classicità (Platone, Euripide, Giove) che causa l'inondazione dell'onnipresenza del coefficiente visivo, da cui si sviluppa la narrazione:

Gli occhi non guardano né insieme né contemporaneamente. Lo sguardo se lo scambiano. Le due pupille non s'incontrano mai sopra lo stesso punto. Lavorano a vicenda: una per volta [...]. Mentre uno guarda, l'altro riposa [...]. Perché se il riposare di un occhio mentre l'altro guarda è proprio di tutti gli uomini [...] senza nemmeno accorgersi di guardare [...] a coloro di cui lo sguardo sfiora, scorre scivola ingenuamente: ben maggiore essa è in quanti il guardare costituisce un'operazione illuminata consapevole, un atto che suscita commozioni mentali, un esercizio richiesto dal voler esaminare, indagare, scoprire [...]. Penso all'espressione un poco distratta e trasognata degli strabici. Penso all'indefinibile fascino dello sguardo storto [...]. Ricordo un ritratto di Platone con lo "sguardo doppio", sebbene la pupilla non fosse segnata. Ricordo la pupilla deviata di Euripide. Di colui che per primo fra i tragici greci ebbe il sentimento chiaro e consapevole dell' avventura e del viaggio [...]. E penso che nelle statue di Giove, e comunque delle divinità olimpiche, sempre si ritrova lo sguardo doppio. (Tordi 143)

Indizi consistenti lasciano supporre che l'analisi della tematica dell'occhio sia culturalmente suggerita dalla grecità dell'autore. La terra in cui si nasce plasma sempre, come è naturale, la forma mentis di ogni individuo: ma niente quanto l'intrigo e il mistero delle costanti immaginative greche influisce così profondamente sulla vita: "la terra sulla quale l'uomo vive trasforma l'uomo a sua propria immagine [...]. S'intende che non tutti i luoghi geografici hanno una eguale virtù assimilatrice, e la terra greca è certamente molto più forte" (Savinio, Opere 97-100).

Similmente a Hermaphrodito, Casa "La vita" (1943) si (ri)struttura intorno a episodi, non collegati fra loro tematicamente, ma suddivisi da un brevissimo proemio, a forma di cantilena ritmata per bambini, intitolato "Occhio" e numerato in ordine progressivo. Alcuni "Occhi" fluiscono anche da una suddivisione interna (per esempio l'“Occhio 3" si prolunga in variante e seconda variante). Si deve insistere sul significato attribuito allo sguardo nell'economia strutturale del romanzo che scandisce tutto il contesto narrativo come iter visivo. Il libro si apre così: "Bell'Occhio guardatore / Sali anche stamattina / In cima al tuo bel fiore / $\mathrm{E}$ cantaci la brina / Cantaci la mina / cantaci l'ondina / Canta l'aspirina / Al mal del nostro cuore"; e si conclude in maniera logica: "Qui l'occhio si chiude". Sembra, pertanto, che l'intero testo scaturisca da una visione, una "salita" seguita da una "chiusura" dell'occhio che immerge i lettori nel flusso della immaginativa classica, extratemporale, continua, onnipresente. Ma anche qui non siamo nell'ambito del surrealismo fantastico come libero volo della fantasia, tramite "l'écrituire automatique", ma davanti all'elaboratezza di scenografie mitologiche (per esempio il tema della meta- 
morfosi in "Flora") che con poteri magici dissolve l'evidenza del quotidiano. L'arte non assume valore nella capacità deformativa del reale, ma viene concepita come costruzione e lettura del passato. L'equivalenza fra infanzia e grecità prende corpo anche in altri testi, fra i quali basti citare Tragedia dell' infanzia (1937) e Infanzia di Nivasio Dolcemare (1941). In Casa "La vita" sin dal primo capitolo, "Alla città della mia infanzia dico", la categoria della nostalgia diviene una chiave di lettura fondamentale per l'intero testo in quanto Savinio ripercorre la formazione classica e il suo rapporto di simbiosi, da cui ostinatamente si sforzerà di non uscire mai, con la "grecita":

Nulla è tanto propizio all' animo appassionato e curioso dei bambini, quanto gli aspetti colmi e misurati quali amavano e produssero gli antichi della terra dov'io nacqui [...] ben fortunato mi reputo di essersi formata laggiù la mia ragione, fra i templi portatili, le colonne che girano assieme al girare del sole, le statue animate di serena magia [...] sempre mi riappare nel fondo [...] la città della mia infanzia. Sorge dalla mia nostalgia inestinguibile, si leva come lo spettro dolente e crucciato della sola felicità che la vita mi ha largito. (17-19)

Il passo che segue è estremamente importante in quanto collega la memoria della letteratura antica (il mito di Orfeo) ai percorsi visivi artistici dell'autore. L'unita concettuale, che ho fin qui tentato di dimostrare fra le varie sezioni della ricerca, si palesa allora chiaramente. Tutti i motivi ricorrono insieme e vengono armonizzati in una concezione dell'atto poetico generato dall'oscillazione perenne, da una vista superiore. Mi spiego meglio: le riflessioni sul mito di Orfeo teorizzano un organismo poetico che partecipa dell'essenza di quella stessa storia mitologica:

Orfea è un errore. Ma è veramente un errore? In Orfeo e nella sua leggenda è qualcosa di ambiguo. Tra il sì e il no. Quel tra sì e no che è la condizione umana di là dalla categorica determinazione dei sessi: di là dalla stessa determinazione del divino e dell'umano. Regno di Ermafrodito. Suprema maturità. Orfeo un professionista dei misteri, e come tale non poteva limitarsi a vedere un solo lato delle cose, come gli uomini soltanto uomini, le donne soltanto donne. Certe professioni richiedono libertà di passaggio [...] da sesso a sesso, come insegna Tiresia. Orfeo fece per Euridice ciò che ogni marito farebbe per la propria moglie [...] ma si voltò a guardarla. (Opere 798)

L'osservazione di percorsi surreali rinnova, gratifica e stimola l'artista, con la delega a quello sguardo privilegiato che lo indirizza a palesare l'al di là del dato dell'esperienza umana e della morte. Il binomio classicità-grecità (Orfeo e memoria) viene mescidato con la moderna e romantica esigenza di movimento:

di nulla si giova tanto l'intelligenza, nulla l'affina tanto, quanto il raffronto tra aspetto e aspetto di una medesima cosa, quanto la rivelazione del lato intimo e nascosto delle 
cose. E' il rovescio della medaglia che fa progredire il mondo [...]. Respingono gli Italiani l'esistenzialismo, respingono il romanticismo, respingono ogni sguardo interno. (Scatola Sonora 69)

\section{La morte e la metamorfosi come (ri)nascita al passato}

Nell'analisi fin qui esposta, l'immagine dell'Ermafrodito si è imposta quale metafora del desiderio costante di osservare e riprodurre gli opposti. L'Ermafrodito, ora, diventa resa metodologica della necessità della duplicità dello sguardo: di quella capacita visiva particolare, che conduce alla conoscenza del non visibile, delle possibilità combinatorie e più complesse della vita. Rimane da verificare il momento e la maniera in cui si scopre, "l' altro aspetto delle cose" tramite la verifica dell'espressione mitologica della metamorfosi. Dallo studio attento di numerose pagine, colgo una connessione attuata da Savinio fra questo fenomeno e quello della morte: spesso le persone defunte ritornano sotto nuove sembianze (anche in pittura), in quanto la morte designa la conclusione di un'esistenza e l'inizio di una nuova forma di espressione. La lettura di due racconti di Casa "La vita" preciserà tale interpretazione.

Il pensiero della morte accompagna l'arte di Savinio costantemente, spesso rielaborato con considerazioni demistificanti sulla storia, sul passato che annienta ogni azione umana; fino alla conclusione estrema proclamata in Dico a te, Clio: "la vera meta della vita: sparire". Nella prefazione a Casa "La vita", Savinio guida ad una lettura dei racconti in termini della loro affinità tematica. La centralità dell'idea della morte, che egli dice accompagnarlo nei suoi pensieri da sempre, spiega il romanzo precedente Narrate o uomini la vostra storia (1942). Vi si riferisce di conclusioni umane "vere", di uomini "capaci di morire", una ricostruzione storica di alcune esistenze fino al loro "guizzo" finale: il salto nell'altro stato, appunto, quello della morte ("la morte di Gemito è appena il guizzo di un delfino sul mare"). Nel racconto "Mia madre non mi capisce" (in Casa "La vita") la morte viene precisamente a coincidere con un "passaggio" attraverso una piccola fessura: ${ }^{15}$ tale balzo pud indicare non soltanto la morte intesa come sparizione dell'individuo, ma anche la sparizione dell'individuo sotto quelle vesti di cui si è narrato finora e la sua rinascita in sembianze altre. La metamorfosi e la morte in realtà riproducono uno stesso fenomeno, ora ce lo presentano per intero, ovvero concluso, ora, invece, illustrano nuove possibilità combinatorie dell'esistenza. Proprio così percepisce la morte il Signor Münster, protagonista del racconto dal titolo omonimo, in Casa "La vita": una nascita cosciente alla ricerca "del meccanismo intimo" della vita. Questo si rivela di estremo interesse per ribadire la concezione formativa dell'arte che distrae dalla morte come nullifi- 
cazione generale del tutto, secondo una lente visiva diversa da quella normale. E, infatti, si è definita la costante strutturale di Casa "La vita" (e di Hermaphrodito) nella presentazione di episodi, per così dire visivi. Si ristabilisce l'usuale fluidità del pensiero di Savinio che oscilla fra la memoria classica che promuove la ripresa di racconti mitici, quale quello della metamorfosi, e la sensibilità tutta romantica verso l'esperienza della morte:

Romanticismo è il sentimento della continuità della cosa al di là dalla cosa; all'infinito [...] l'anima non si stacca dal corpo [...] meglio il corpo per virtù di quella parte di sé che chiamiamo anima, si prolunga all'infinito [...]. Romanticismo è questo senso di infinito delle cose [...] al di là di ogni meta [...] insensato. Il sentimento romantico è un invito a morire [...]. In altre parole scopre lo staio infantile dell'uomo [...]. Farsi adulti significa negare quel [...] profondissimo desiderio di morire, ossia ritornare [...] all'infanzia. (Opere 1065)

Se procediamo in ordine logico si giunge al parodosso della coincidentia oppositorum: se romanticismo rimanda qui al sentimento dell'infanzia; se in altri passi citati è invece la classicità a equivalere alla riappropiazione del passato, anche individuale, coincidendo col percorso biografico; allora il romanticismo si innova nel classicismo, il romanticismo diventa grecità e il classicismo modernità, nell'invito a non uscire mai dall'infanzia. Ma è ancora al testo che dobbiamo rivolgerci per scoprire ulteriori nodi della levità saviniana.

I motivi (romantico) della morte e (classico) della metamorfosi informano di sé i due racconti: "Il Signor Münster", "un morto-vivo" che alla fine perderà completamente il controllo sui propri arti e assisterà con distacco alla propria frantumazione, e "Flora", una statua che si anima. Il primo racconto presenta un personaggio cosciente del proprio processo di trasformazione da vivo a morto. Nella consapevolezza ormai di aver capito, egli assapora il proprio stato di "grazia", fino al travestimento femminile e all'uscita notturna nei nuovi abiti. Questa metamorfosi finale gli permette di incontrare l'Aurora, vecchia, discinta, scarruffata, reduce da una notte di bagordi a casa dell'Ingegner Titone, che si affretta a far sorgere il nuovo giorno. Nel tentativo di fermarla, ogni arto del Signor Münster si frantuma per la strada: "Un mucchio di stracci rimane per terra. Il cappello di paglia ruzzola per conto suo, come la ruota di un carrozzino da bambini. Il mantello continua a palpitare [...]" (Casa 286). Il racconto si costruisce, dal punto di vista grafico, con corsivi e note. Fra queste, l'ultima scioglie e completa il nucleo della narrazione nella conoscenza acquisita dell'al di là della morte, dell'inspiegabile presenza di una surrealta: "Il caso del Signor Münster [...] la prima, sola, vera testimonianza che nell'uomo anche un'altra cosa vive oltre il corpo, e sopravvive alla morte del corpo [...] gli consentì [il vedersi frantumato] di 
scomporre gli aspetti delle cose e di scoprire gli elementi che li costituiscono; una maniera che gli mostro la vita non come apparenza ma come meccanismo intimo; una maniera che gli rivelo [...]" (287).

Il motivo dello sguardo e della morte si sovrappongono nella ripresa della figura classica di Medusa, colei che pietrifica chi la guarda. Anche a questo proposito Savinio da una parte ricorre alla classicita, ribadendo la sua totale impermeabilità ai richiami della cultura antica:

Il Signor Münster, che ha sempre avuto un grande amore per la mitologia greca, si pensa in questo momento in istato di Medusa, e come se la vista di se stesso possa farlo morire: far morire lui che è già morto. (273)

Dall'altra esce dal perimetro di quella costruzione tradizionale mitologica e afferma una sostanziale autonomia:

Sulla Medusa, Ettore Romagnoli aveva insegnato anni sono al Signor Münster un particolare che il Signor Münster ignorava, e che si trova in Pindaro. Dice questo poeta che ogni mille anni la Medusa dimette per un giorno la sua natura di mostro, e ritrova quella di vergine bellissima; e durante questa giornata di rigenerazione e nella notte che la segue, essa canta un canto sovrumanamente dolce e infinitamente triste; e a udire quel canto anche i mostri si placano nelle foreste e stanno attoniti ad ascoltare. (273)

Non stupisce, quindi, che il nuovo percorso visivo del protagonista volga indietro e colga l'inaccessibile allo sguardo normale nel recupero delle divinità olimpiche del passato:

Il Signor Münster entra in stato di grazia. Ora i suoi occhi vedono ciò che finora non avevano mai veduto, ciò che nessun occhio umano ha mai veduto, e solo una percezione straordinaria, l'ultima e più acuta consente di vedere: ciò che vede l'occhio di un santo, di un facitore di miracoli, di un dio in efficienza - ciò che vede l'ultimo sguardo di uno che muore ... . Vede ... Vede ... Vede ... lo sguardo del Signor Münster si acuisce anche più. Vede più lontano. Vede più profondo. Vede più antico. Vede Giove seduto, con la barba turchina e gemmata di chiocciole, e i suoi fulmini di gesso dorato raccolti in grembo. Vede Mercurio che per diporto vola ancora in obliquio, reggendo a braccio teso un bastone e fingendo di recapitare delle lettere. Vede altre divinità ridotte a forme pallidissime, e a quelle non riesce a dare nome. $\mathrm{E}$ dietro $[\ldots]$ altre forme ancora [...]. Anche l'orecchio del Signor Münster si è fatto straordinariamente acuto [...]. (280)

La naturale conclusione avviene nella "Postilla a 'Il Signor Münster'", in cui il protagonista riconosce la verità ultima sulla dimensione temporale dell'esistenza ("un equivoco o più semplicemente errore di ottica"); ne consegue l'abolizione della distinzione fra le categorie del futuro, presente e passato: ${ }^{16}$ 
Scoprendo questo rovesciamento di posizioni, il Signor Münster capì finalmente quale specialissimo riguardo gli aveva usato la sorte offrendo ai suoi occhi di morto quella magnifica visione del passato, ossia la visione dell'avvenire nel quale egli era per entrare; e terminò la sua morte nel sentimento ineffabile felice di una nascita cosciente e che l'uomo si è scelta da sé. (293)

Leggiamo adesso l'altra storia contenuta in Casa "La vita", nel tentativo di ricollegarne la lettura alle considerazioni sin qui svolte. In "Flora" il tema mitopoietico della metamorfosi costituisce il leit-motiv fantastico del racconto, catalogato sotto la filastrocca "Occhio N. 2" di sei versi ritmati, cui segue anche una rielaborazione fantastica in prosa, non gratuita né nell'economia tematica della storia (ambientata nei suoi momenti essenziali in un bosco), né in quella generale dei viaggi 'marini' saviniani. ${ }^{17}$ L'incipit del racconto è piuttosto realistico, descrive il dolore nel protagonista, Marco, in seguito alla fine della relazione con Edmea, fino alla fuga dalla città e al rifugio in casa dell'amico Valerio. Qui, insieme alla nuova ambientazione geografica, improvvisamente scatta un tono nuovo della narrazione che dirige al senso di estraneità, al distacco del personaggio ("I dolori non riuscivano a dominarlo [...]. Delle fanciulle aveva un'idea mitica"). Marco alla ricerca di una dimensione altra, di suoni e fantasia, approda ad una realtà "orfeizzata", grazie alla nuova capacità di sentire, di calarsi nel regno classico: "nulla gli sfuggiva dei rumori minuscoli che, tutti assieme, compongono il silenzio della natura". E incontra Flora, una statua che si anima:

Marco, tremante di commozione, fissava la statua che si animava a poco a poco. Ascoltava quella voce opaca, velatissima; quella voce che veniva di lontano, dal cuore della pietra [...]. Gli occhi di Flora brillavano più vivi, le sue labbra sbocciarono in sorriso. Era dunque lui, uomo, che aveva operato quel miracolo [...]. Flora raccontò a Marco la vita misteriosa delle statue; le quali non sono materia inanimata come crede il volgare, $m$ a creature vive e poi imbalsamate in pietra. Ov'esse abitano per sempre in compagnia della loro anima e dei ricordi della loro vita mortale. (82)

Così l'occhio di Marco diventa in grado di scoprire al di là delle apparenze, nel momento in cui si "disumanizza". L'elemento fantastico, all'inizio tangenziale al racconto, si costruisce su similitudini o echi mitologici e si allarga sia in una dimensione cronologica, perché Marco d'ora in poi si rifugerà nel bosco ogni sera, sia in una dimensione spaziale, in quanto nel parco Marco "seppe che là, in quello stesso parco, c'era il padre e la madre di Flora, statue essi pure, e una zia" (83).

In entrambi i racconti si attua la fusione fra uomo e natura proprio nel momento della trasformazione dalla vita alla morte. In "Il Signor Münster" il protagonista "morto-vivo" sente l'esigenza di "avvicinarsi alla natura e immergersi in essa, per ritrovare, assieme con quella non più promessa ma certezza di amore, anche quel remoto e misterioso tepore, quel sonno dolcissimo 
e così vicino al risveglio, il cui ricordo ora nitidamente gli ritorna dai tempi che hanno preceduto la sua nascita". Parallelamente Marco in "Flora" "si orfeizzava". Evidentemente, se riflettiamo in termini generali, esiste una memoria ovidiana delle metamorfosi. In Savinio, come in Ovidio, alla fine si assiste ad una sorta di risarcimento in un nuovo rapporto raggiunto fra $\mathrm{i}$ due esseri appartenenti ormai a mondi diversi. È una nuova nascita, in armonia con la lettura di Münster della morte: "un sentimento ineffabilmente felice di una nascita cosciente". Ma in Savinio la metamorfosi va in senso inverso rispetto al grande scrittore classico: cio che in Ovidio era uomo o donna diventa inanimato per fuggire catastrofi, si pensi a Dafne che per mantenere la verginità dalla passione di Apollo viene trasformata in albero o Siringa diventata canne per sottrarsi alle insidie di Pan. In "Flora", invece, Marco, ormai estraneo alla realtà "umana" quotidiana, si affida lui stesso e per sempre all'altro mondo tramite una morte-metamorfosi:

[...] e nel cuore scoprì finalmente il moto appena percettibile di un respiro tenuissimo, l'arcana freschezza di un'anima nascente, il palpito di un cuore che dal fondo del silenzio, dal fondo dell'inerzia, dal fondo dell'oscurità cercava a poco a poco, casto e fidente, di riaffiorare alla vita. L'intero universo irruppe in quel momento nel petto di Marco. Si riaccesero i paradisi spenti, e le luci che l'avvenire tiene in serbo per le felicità future, brillarono di colpo e tutte assieme. E Marco, pieno d'immenso amore, penetrò nell'ombra degli alberi. (87)

Ho detto di Ovidio nell'economia generale e classica della scrittura saviniana; alle metamorfosi ovidiane viene anche dedicato un racconto nello stesso testo, dal titolo "Le nuove metamorfosi di Ovidio". Tuttavia, come accennato all'inizio di questa ricerca, la sensibilità saviniana deve aver tenuto in mente la letteratura fantastica della generazione precedente, in particolar modo quella francese, considerata anche la capacità del Nostru di lettura in originale. In questa direzione il modello più diretto di "Flora" appare "La Vénus d'Ille" di Mérimée, composto nel 1834. La ripresa savinana annulla il carattere noir del racconto francese di una statua di bronzo, scoperta per terra, che provoca danni inumani a chiunque le si avvicini: spezza la gamba di chi cerca di innalzarla sul piedistallo; scaglia pietre contro poliziotti; uccide il protagonista figlio M. Alphonse, durante la prima notte di nozze; uccide il padre; e anche quando viene fusa in campana continua ad esercitare i propri poteri diabolici ("Mais il semble qu'un mauvais sort poursuive ceux qui possèdent ce bronze. Depuis que cette cloche sonne à llle, les vignes ont gelé deux fois"). Così si conclude la narrazione. In Savinio la macchina narrativa si sviluppa inizialmente su pretesti contrari: si assiste ad una separazione amorosa (mentre nel testo francese si celebra un matrimonio), ad una partenza dalla città alla campagna del protagonista (contro lo spostamento da Parigi alla Catalogna del protagonista francese). Tuttavia, la fondamentale differenza fra 
i due testi riguarda l'atmosfera che si crea intorno alla presenza della statua. In Savinio Flora si svela per tutto il racconto esclusivamente al singolo protagonista: "Ma perché solo a lui era stata rivelata quell'esistenza straordinaria?". Flora "suggeriva doveri di pudicizia, gl'ispirava idee di profonda onesta" e rappresenta per Marco la felicità della dimensione non umana, che io chiamerei "mitologica". È il protagonista stesso che risponde al richiamo della statua e ne desidera la simbiosi, "pieno d'amore". La statua che si anima rappresenta il privilegio di quella vista estrema cui tante volte ho ormai accennato in queste pagine. Una dimensione di mistero e di surrealtà che si manifesta soltanto a chi è in grado di apprezzarne la meraviglia, in questo particolare racconto a colui che ama. La statua in Merimée, invece, è una Medusa, visibile a tutti: "Elle a l'air méchante [...] et elle l'est aussi" e incute paura al solo osservarla: "Elle vous fixe avec ses grands yeux blancs [...]. On dirait qu'elle vous dévisage. On baisse les yeux, ou, en la regardant [...]". Colpisce la sua bellezza infernale, la maestosità crudele, la maliziosità severa:

Quant à la figure, jamais je ne parviendrais à exprimer son caractère étrange [...]. Ce n'etait point cette beauté calme et sévère des sculpteurs grecs [...] ici, au contraire [...] la malice arrivant jusqu'à la méchanceté. Tous les traits étaient contractés légèrement [...]. Dédain, ironie, cruanté, l'absence de toute sensibilitée [...] quelque chose de féroce [...]. Cette expression d'ironie infernale [...] faisait baissez les yeux à ceux qui la regardaient. (448)

Il finale permette di stabilire un contatto diretto fra le due narrazioni. In entrambe la morte del protagonista viene messa in relazione alla presenza della statua. Ma ciò che maggiormente importa è la posizione identica dei due cadaveri. Marco viene trovato esanime nelle braccia della statua, incastrato sotto la mano destra di Flora, rialzata al petto per sorreggere la gonna colma di fiori. La posizione della mano sinistra della statua francese, immobile sul piedistallo, è identica, ma quello che la vedova racconta, a proposito della morte del marito, è spaventoso. In entrambi i testi fra il protagonista e la statua sorge un forte legame sentimentale, ma mentre per Marco diviene scelta cosciente e gratificante ("Il pensiero di Flora gli dava una commozione di bene"), Alphonse diviene vittima della "Vénus", per averle infilato all'anulare, durante una partita a palla, la propria fede matrimoniale. Nel tentativo disperato di recuperare l'anello la statua ritira il dito e si considera ormai la sua sposa. E il testo si avvia alla fine, tramite la narrazione della giovane vedova, ritenuta impazzita dal dolore per sostenere di aver assistito all'omicidio del marito da parte della statua:

Elle était couchée [...] depuis quelques minutes, les rideaux tirés, lorsque la porte de sa chambre s'ouvrit et quelqu'un entra. Alors madame Alphonse, [...] la figure tournée vers la muraille [...] persuadée que c'etait son mari [...] le lit cria comme s'il 
etait chargé d'un poids énorme. Elle eut grand peur, mais n'osa pas tourner la tête [...]. Puis elle fit un mouvement involontaire [...] et elle sentit le contact de quelque chose froid comme la glace [...]. Peu après la porte s'ouvrit une seconde fois, et quelqu'un entra, qui dit: "Bonsoir, ma petite femme" [...] on tira le rideaux [...] le personne qui était dans le lit, à côté d'elle, se leva sur son séant et parut étendre le bras en avant. Elle tourna la tête alors [...] et vit son mari à genoux auprès du lit [...] entre les bras de la Vénus de bronze [...] sans mouvement. (464)

Sul cadavere di Marco "infilato sotto il braccio di Flora" Savinio, invece, lascia aperta l'interpretazione; in ogni caso non si fa presupporre l'iniziativa crudele della statua, dato il lamento finale (in latino, come nel testo francese) di dolore di Flora:

Infine anche l'assiolo tacque, e la notte alta e pura non fu abitata più se non da quella voce lacerata da un immenso dolore, che dal fondo delle montagne, dal cuore della pietra continuava a chiamare "Marce! ... Marce! ... Marce! ...". (89)

Tener presente la fonte francese serve a definire il punto esatto dove poi il nostro autore la sostituisce con materia diversa: il motivo dell'animismo di una statua viene assorbito da Savinio in una dimensione più ludica, leggera, meno tragica. La dimensione noir è sparita completamente dal racconto di Savinio che piuttosto attribuisce connotati negativi non alla statua vivente, ma alla fidanzata di Marco, dalle labbra paradossalmente "gelide", così descritta mentre dorme: "I capelli corti e nerissimi sparsi sul guanciale, davano alla sua testa piccola un che di serpentinamente cattivo. L'ombra delle narici si mangiava il naso brevissimo, segnava in mezzo alla faccia il triangolo nero del teschio [...]". Il finale del racconto Flora non prevede, comunque, un omicidio da parte della statua animata, ma una scelta cosciente del protagonista di calarsi nell'aldila del mistero. Queste pagine sottolineano, ancora una volta, l'esistenza di una surrealtà che tiene a mente certe immagini classiche in quanto si svela a chi bene sa osservare: al protagonista-autore che, appunto, è un Orfeo. ${ }^{18}$

\section{La conclusione del viaggio con Savinio}

Affiora dalle pagine saviniane la necessità di afferrare e completare un quadro sempre più ricco e imprevedibile dei fenomeni. Questa esperienza presuppone una "spersonalizzazione" (Cirillo 106), disumanizzazione, frantumazione del protagonista. La metamorfosi e la morte, come fenomeni simili e atti supremi, sanciscono definitivamente la rottura del legame col mondo umano e quotidiano, tramite il recupero della dimensione mitologica. Le testimonianze sono numerose e note al lettore di Savinio. Qui sono stati presi in 
esame la frantumazione del corpo di Münster e 1'“orfeizzazione" di Marco; accenno, fra gli altri esempi, al restringimento della figura della "grande Signora Dolcemare" (a noi ben nota anche da altre pagine di Infanzia di Nivasio Dolcemare) e alla sua apparizione al figlio Nivasio in forma di gallina "piccola piccola" in "Mia madre non mi capisce" (Casa "La vita"). La dimensione surreale provoca ciò che individuerei come metamorfosi a catena: la madre-gallina dialoga col figlio che, ecco l'altra sorpresa fantastica, assume a sua volta le sembianze di un pulcino nella fine del dolore e nel recupero del passato. Anche qui, metamorfosi e morte diventano vicende analoghe e comunque complementari.

Per sciogliere ulteriormente il nodo di questi "passaggi", propongo di ricondurli all'esperienza del viaggio e della mobilità che Savinio auspica come regola di vita: "Il dio greco è passeggero. È questa la sua migliore qualità, il suo Sommo bene [...]. A imitazione di Dio, anche l'uomo immobile è pericoloso, per il bene dell'umanità, consiglio lo stendhalismo come regola di vita" (Opere 32). Il tema del viaggio dà il titolo all'ultimo racconto di Hermaphrodito, "La partenza dell'argonauta", che ho analizzato in altra sede. Me ne servo qui per riprendere la conclusione sull'interiorità di quel cammino ed applicarla ad altri avvenimenti che, all'apparenza, non sembrano all'esperienza del viaggio direttamente collegarsi: appunto la metamorfosi e la morte. E questo perché sia nel percorrere viaggi che nel rappresentare l'enigma della metamorfosi e della morte si assiste di volta in volta ad un'indagine svolta nella memoria del passato culturale e biografico. Ho dimostrato la pregnanza del richiamo al mito di Orfeo da parte di Savinio, sia in sede narrativa (in "Flora" il protagonista "si orfeizza") che in sede giornalistica. Per avvalorare l'equivalenza già stabilita (fra il mito di Orfeo e la poetica di Savinio), direi che come Orfeo, sempre vigile nel cogliere "di là della stessa determinazione del divino e dell'umano", scende a recuperare Euridice "ma si volta a guardarla", così l'autore sprofonda nell'immaginario mitologico, e si ferma a guardarlo in una luce diversa: quindi, ne coglie il mistero e lo spalma di modernità, tramite la chiave ironica. La predilezione per i viaggi tutti interiori, alla ricerca del passato, nel mare "amico dell'infanzia" se vede la sua prima formulazione nella sezione finale di Hermaprhodito (grazie alla similitudine protagonista/Giasone) si prolunga nei testi e/o racconti successivi, secondo quella che indico qui come creazione a conseguenza. Da Hermaphrodito, seguendo la chiave di lettura dell'autore stesso in "Piccola Guida alla mia Opera Prima", sono approdata a Casa "La vita". Si tratta di itinerari all'insegna del recupero delle fonti che spesso si risolvono in un viaggio geografico verso la Grecia, attraverso il mare. È l'esempio delle pagine intitolate "Walde Mare" in Casa "La vita" che descrivono un viaggio fantastico tutto svolto nella memoria del passato, non solo dell'individuo-protagonista che "serba i ricordi marini dell'infanzia", ma anche della collettivita occidentale, 
dati gli incontri con le (ormai note) divinità marine sui generis. Il racconto descrive Messanio, ospite dei Dolcemare, che il Signor Visanio, padre di Nivasio, chiama "mostro marino", quale essere a metà fra uomo e pesce. Siamo di fronte alle usuali occorrenze: una partenza dalla terra al mare, una metamorfosi marina, un meraviglioso tutto di origine mitologica, un processo di antropomorfizzazione del mare stesso. ${ }^{19}$ Ed ancora una volta Hermaphrodito fa da guida. Nel capitolo finale del romanzo, avevo già segnalato come l'arrivo in Macedonia comportasse una sorta di regressio ad uterum, un ritorno alla grecità ("[...] mi riviene la figura della madre che richiama i figli sul suo corpo e sulla sua carne") e all'infanzia ("il mare che ritrovo è quello stesso che mi fu amico insino dal mio nascere terrestre) ${ }^{20}$

Alla luce del dopo Hermaphrodito, appare evidente che il messaggio riproduce sempre se stesso, assorbito in nuove strutture narrative, in uno sperimentalismo linguistico meno incessante, ma che conferma la costante volontà di afferrare l'impossibilità mitica passata, immagine della perfezione degli opposti: nell'ipotesi di un'idea della modernità basata sull'idea nietzschiana dell'eterno ritorno con un recupero testuale di antichi modelli, ma non per questo priva di agganci nella contemporaneità, in un rapporto di dialogo e polemica con l'avanguardia novecentesca. Savinio ci ha talmente educati al gioco con i contrari, al punto da essere tentati, proprio in chiusura, di assemblare insieme le sue riflessioni, fino ad estenderle in una formulazione ossi-morica: sembra lecito leggere i percorsi saviniani quali viaggi romantici verso l'infinito classico?

\section{The Johns Hopkins University}

\section{NOTE}

1 Perla lettura diretta di testi greci e latini si vedano gli articoli "Anche dell' amore c"è una specie di ammasso" (in cui si cita Omero e Euripide come fonte per la rielaborazione di Capitan Ulisse e di Alcesti di Samuele) e "Il refuso si vendicò sulla tomba del pedante". Opere 875-79 e 987-90.

2 Savinio ribadisce la differenza fra la propria poetica e quella per esempio dell'estetismo dannunziano del culto della parola: "Metafisica e regia". Opere 955-58.

3 Si veda Carlino, "A proposito" 21-29. Si evidenziano qui indizi consistenti che lasciano supporre la conoscenza di Savinio degli Chants de Maldoror in Hermaphrodile di Lautréaumont pubblicato in francese in Lacerba 15 (1913).

4 Fontanella ha parlato di linguaggio aleatorio, soggetto ai casi, guidato dall'immaginazione e dal meraviglioso in Savinio, analizzando il primo romanzo, Hermaphrodito: "una sorta di fantasticità a occhi aperti, il sogno che esplora il disordine di sé e dell'universo" (50). Il critico legge anche Angelica o la notte di maggio (1927) in direzione "dell"humus (ludico) surrealista [...] in uno stato di deambulatorietà della scrittura, ovvero d"immaginazione permanente" (127).

5 Più tardi Savinio rifletterà in Dico a te, Clio: "Freud è morto, e sul suo conto ne abbiamo sentite qui in Italia di cotte e di crude [...]. Per noi, dopo Schopenhauer, Freud è il migliore degli educatori. Anche il passo grave e pacato della sua prosa è un passo da educatore. Freud mostra le cose della sua vita, "tutte" le cose, anche le più sepolte, ma non le giudica: lascia all'allievo libertà di prendere o lasciare. Educare, come dice la parola, non deve essere altro che "guidare"' (120). 
L'interpretazione dei miti venne usata in psicanalisi, per stabilire la presenza dell' 'individuo al di là della storia. Per il fascino esercitato dalla psicanalisi si veda anche la recensione di Savinio al libro di Cesare Musatti in "Perché noi ltaliani non amiamo la psicanalisi" (Opere 1063) e al testo di Jung in "Luna a destra" (973). Per una ricezione e messa in discussione della psicanalisi in letteratura si consideri il romanzo Angelica o la notte di maggio. Nella trascrizione "modema" della favola di Amore e Psiche (che qui diventa una ballerina greca, prostituta con la quale il protagonista non riesce mai a consumare le nozze perché essa dorme sempre a occhi spalancati) si mettono in discussione la psicanalisi, che non riesce a guarire il barone, la famiglia, luogo di sofferenza e sfruttamento, i rapporti umani, basati sull'incomunicabilità e pertanto non veri. Di Savinio compare anche una litografia dal titolo "Angélique o la nuit de mai" ( $\mathrm{cm}$. 28x22,7), incisa per una progettata edizione francese del romanzo che doveva essere illustrata da dieci litografie dell'autore. Non mi risulta che tale edizione sia stata ultimata.

6 Anche fra le liriche di Apollinaire, in "Zone" e "La Chanson du Mal Aimé" (nella raccolta Alcools del 1913) sono individuabili precise immagini del testo saviniano "Les Chants de la mimort" pubblicato nel 1914 a Parigi in "Les Soirées de Paris". Mi riferisco sia all'atteggiamento del poeta in uno stato di angoscia e desolazione offerto in "Zone" che rimanda a "Chants de la nuit" con cui si chiudono "Les Chants de la mi-mort" (un uomo solo piange di fronte al vuoto e all'incomprensione da cui è circondato); sia alla riproduzione dell'espressione grottesca de "La Chanson du Mal Aimé" di Apollinaire: "Ta mère fit un pet foireux et tu naquis de sa colique", in un quadro saviniano in cui la colica matema diviene fenomeno di gioia per l'universo. $<\mathrm{On}$ entendit alors une longue éructation de joie, / et la planète péta; [...] "qu'est-ce [...] ?" "Un dieu souffre [...], / ou bien l'Universe s'abîme [...]" dit l'Onirocrite sur l'autre péninsule [...] \> . Il rapporto "letterario" fra Savinio e Apollinaire si legge, fra l'altro, nell'episodio "La figlia dell'imperatrice" in Casa "La vita", in cui Savinio scrive di aver appreso a memoria il componimento "Zone" e di averlo recitato in occasione della prima visita all' amico al Boulevard Saint-Germain. Rimando anche alla continuità fra i personaggi de "Les Chants de la mi-mort" $e$ quelli dei primi due capitoli composti in francese di Hermaphrodito dal titolo: "Prélude Tête antichambre de ministre" e "Drame de la ville méridiane", soprattutto in relazione al primo, secondo, terzo e ultimo quadro di "Drame". Si veda Ostenc.

7 Tra i contributi più significativi relativi alla soluzione linguistica, Roscioni, Carlino, Alberto Savinio, Pedullà, Scrittore ipocrita.

8 Così a proposito di parodia e evocazione mitica, Jesi interpreta: "Pure, chi compie una parodia sembra, in fondo, non potersi staccare dall'oggetto parodiato: una forza lo induce [...] a passare oltre; un'altra forza gli impedisce di ricacciare quell'oggetto nell'oblio e nel silenzio. Essa comporta un volgersi indietro, se pure contrastato e polemico; e nel vincolo che unisce il parodista all'oggetto della parodia è lecito riconoscere [...] la sopravvivenza delle figure di un mito con le quali ci si difende, ma che non si possono escludere dalla propria psiche" (15).

9 Quattro furono le recensioni al romanzo: di Papini in "Il Tempo", il 3 gennaio 1919; di Binazzi in "Il Giomale del mattino", il 2 marzo 1919; di Meriano in Ardita, il 15 aprile 1919; di De Pisis, la cui recensione è stata pubblicata per la prima volta soltanto in Documenti ferraresi (in Catalogo della mostra di Ferrara). Papini scrive nella recensione: "[...] non è facile dare a quegli altri un'idea di questo libro che ha nel titolo stesso un avviso di ambiguità [...] non è un romanzo, benché vi siano più avventure di un romanzo; non è un libro di poesie [...] non è neppure un diario di bordo [...] e neanche una scompigliata sacchetta di frammenti [...]. Un pezzo comincia in italiano e seguita in francese; isolotti di versi s'avvistano in mari distesi di prosa; i dialetti, la lingua franca, il greco, lo spagnuolo [...]" (ora in Papini 715-20). Fra gli ultimissimi contributi, sugli antiromazi saviniani, ho appena letto Stefano Giovanardi, "Repubblica", 24 gennaio 1996: "Ma Savinio non scrisse mai romanzi" in cui si parla di "frammenti, fulminei bozzetti e soprassalti intellettuali. Il paradosso di un neoclassico consegnato all'avanguardia storica".

10 Carlino rivolge attenzione all'imagerie androgina nella cultura del Primo Novecento e ne spiega l'adozione da parte di Savinio in quanto l'ermafrodito si impone quale idea di "totalità estetica" ("A proposito" 27). Roscioni individua il significato del titolo nella "scrittura lambiccata e ambigua" e ne collega la scelta al gusto tutto saviniano per l'equivoco. Filippo De Pisis nella 
recensione al libro scioglie l'enigma del titolo con le due lingue in cui è composto.

11 Il quadro, cm. 35x27, è riprodotto nel catalogo edito da Fabbri, 131. L'immagine saviniana dell’Ermafrodito appare in letteratura nel 1916 e Carlo Carrà la recepisce immediatamente associandola a sue speculazioni formali: il quadro "L'idolo Ermafrodito" (olio su tela, cm 65 x42) apparirà a Milano nella Mostra allestita alla Galleria Chini dal 18 dicembre 1917 al 10 gennaio 1918. Ermafrodito siede sul più alto dei tre parallelepipedi visibili; il colore del primo, verde, nasce dall' accoppiamento degli altri due, che sono in azzurro e giallo. Nello stesso anno nella rivista letterana La Raccolta il medesimo quadro viene riprodotto col titolo "Il dio Ermafrodito". Due anni dopo nell"edizione francese di Valori Plasticill diventa "Le dieu Hermaphrodite".

12 Il quadro, cm. $20 \times 34,5$, è riprodotto nel catalogo edito da Electa, 9.

13 L'altra fonte classica è il Libro 4.310-88 delle Metamorfosi che descrive il congiungimento fra il figlio di Ermes e Afrodite e una ninfa di lui innamorata e da qui la nascita dell'ermafrodito. Per la figura di Tiresia nelle fonti classiche si leggano le Metamorfosi, 3. 320-40; di Sofocle, Edipo re, Antigone; Odissea 11, 12, 13.

14 Moroni analizza quella che chiama "dimensione sacerdotale" in Infanzia di Nivasio Dolcemare, che conduce al superamento degli opposti da pane di un individuo superiore. E cita, come conferma di accorgimenti linguistici che testimoniano del senso di perdita dell'identità, un passo dal romanzo: "Al termine delle vie diverse per le quali cammina l'umanità, Ermafrodito addormentato rappresenta oggi, come al tempo del Simposio, l'immagine ideale della perfezione. Ma non è un dio neutro costui, sebbene il divino totale dei totali. Ed è in nome di Ermafrodito appunto che Nivasio Dolcemare denuncia l'inversione, questa grande solitaria, come nemica della perfezione". Perl'assunto jungiano, Sanguineti 430 e sgg.

15 "E allora Nivasio capi che per passare dalla vita alla morte bisogna curvarsi e farsi piccoli piccoli come per passare attraverso un pertugio molto stretto. Allora Nivasio penso che la morte è il parto più difficile e una terribile nascita. Allora Nivasio sentì che quando la creatura anche più cara a noi agonizza, ossia lotta per il passaggio, quello che essa invoca da noi non è di trattenerla di qua dal passaggio, ma, terribile a dire, aiutarla a passare di là"(178).

16 Anche "Nuove Metamorfosi di Ovidio", in Casa "La vita". È fondamentale segnalare, nell'economia del nostro argomento, che dalla dilatazione del tempo, dall'ostinata volontà a non scindere mai le coordinate spaziali e temporali, Savinio giunge alla "giustificazione" delle favole antiche, in un articolo dedicato a Einstein, il 7 luglio in "Il Tempo" del 1921: "Codeste scoperte si levano come una tardiva ma sicura giustificazione di tutta la mitologia classica. $\mathrm{Ci}$ rivelano perché gli assennati greci avevano collocato i loro dei sulla terra, quasi a portata di mano dei mortali; perché essi avevano assegnato quelle tali limitazioni alle loro divinità, per modo che la potenza di questi dei, quantunque sovrumana, giammai trascenda la misura di una fantasia, per così dire, locale [...]. Ché, a ben guardare, quello che principalmente costituisce il carattere del classicismo, si è la rappresentazione e la continua presupposizione dello spazio in sé (teoria della relatività), che conferiscono quello che di raccolto, dj logico, di saggio, che è come la qualità precipua sia di ogni pensiero sia di ogni opera d'arte classica".

17 "Nel cuore della notte quando tutta la casa dorme, il signor Pietro in preda a violentissimi dolor, è costretto a tirarsi fuori dallo stomaco una intera foresta con le sue querce, i suoi castagni, i suoi lecci, e nasconderla nell' armadio per paura che riponendola sulla sedia accanto alle mutande e ai pedalini, i suoi figlioli la mattina se la portino via per giocare e assieme si prendano gioco del loro papà. Nello stesso tempo, mezz'ora più mezz'ora meno, il Signor Carlo in preda a una tremenda indigestione si deve alzare dal letto e correre al gabinetto scalzo e in camicia da notte, a vomitare il suo mare" (67).

18 Per quanto riguarda l'animismo all'intemo del fantastico novecentesco si legga l'ultimo contributo di Desideri.

19 “Andai ad aprire la porta ... Era il Mare in persona. Il Signor Mare ... Il Mare entrò lentamente e sedé alla mia tavola. Era assurdo e magnifico. Due liquide volute gli nascevano dall'ombelico, si allargavano via via che salivano, e all'inizio delle braccia si arrotolavano in boccoli di spuma, per far spalline. Camminava su due onde inginocchiate, e nel mezzo della faccia glauca, due piccole meduse gli fungevano da occhi" (91). 
20 Che gli spostamenti descritti in "La partenza dell'argonauta" siano essenzialmente interiori è testimoniato non soltanto dalle riflessioni di Savinio contenute nel capitolo successivo di Hermaphrodito intitolato "Epilogo" "Io inclino per natura alle posizioni sedentarie [...] . Io propendo per i viaggi immaginari - quelli che non implicano trasferimenti organici - . Mi pongo dalla parte della commedia e non da quella dell'Odissea. Mi sento attratto verso una terra, una città, una casa - probabilmente perché non possiedo né terra, né città né casa". Ed anche dalla raffigurazione di Carrà nel 1916 di "La partenza dell'argonauta Savinio" che coglie in modo esemplare 1'atteggiamento dell'amico nei confronti del viaggio: Savinio è rappresentato in un interno, immobile nell'architettura del corpo ancorato alla sedentarietà.

\section{OPERE CITATE}

Bramanti, Vanni. Gli dei e gli eroi di Savinio. Palermo: Sellerio, 1983.

Breton, André. Anthologie de l'humour noir. Paris: Sagittaire, 1940.

Carlino, Marcello. Alberto Savinio. La scrittura in stato d'assedio. Roma: Istituto della Enciclopedia Italiana, 1979.

"A proposito di un titolo ingombrante". Mistero dello sguardo. Ed. Rosita Tordi.

Roma: Bulzoni, 1992. 20-29.

Cirillo, Silvana. Casa "La vita" di Alberto Savinio. Roma: Bulzoni, 1975.

Desideri, Giovanella. "Il fantastico". Letteratura Italiana. Torino: Einaudi, 1989. 970-97.

Fontanella, Luigi. Il surrealismo italiano. Roma: Bulzoni, 1983.

Jesi, Furio. Letteratura e mito. Torino: Einaudi, 1968.

Kermode, Frank. The Sense of an Ending. Studies in the Theory of Fiction. New York: Oxford UP, 1967.

Merimée, Prosper. "La Vénus d'Ille". Romans et nouvelles. Paris: Gallimard, 1934. 438-66.

Moroni, Mario. Article not yet titled, forthcoming in The Canadian Journal of Italian Studies.

Nietzsche, Friedrich. Utilità e svantaggi della storia per la vita. Trad. it. Lia Pinna-Pintor. Torino:

Einaudi, 1943.

Ostenc, Michelle. "Savinio et la France jusq'en 1920". Nuova Antologia 2161 (1927): 218-29.

Papini, Giovanni. Scrittori e artisti. Milano: Mondadori, 1977.

Pedullà, Walter. Scrittore ipocrita e privo di scopo. Cosenza: Lerici, 1979.

"Le favole surrealiste di 'nonno' Savinio". La letteratura del benessere. Roma:

Bulzoni, 1973. 335-38.

Raimondi, Ezio. Le pietre del sogno. Bologna: Mulino, 1985.

Ricci, Roberta. "Savinio-Giasone in 'partenza' con gli argonauti". Italian Culture 12 (1994): 189200.

Roscioni, Giancarlo. "Nota a Hermaphrodito". Hermaphrodito. Torino: Einaudi, 1974. 237-52.

Sanguineti, Edoardo. “Alberto Savinio”. Studi sul Surrealismo. Roma: Officina Edizioni, 1977. 405-31.

Savinio, Alberto. Angelica o la notte di maggio. Milano: Morreale, 1927.

Dico a te, Clio. Firenze: Sansoni, 1946.

. Tutta la vita. Milano: Bompiani, 1949.

- Alcesti di Samuele. Milano: Bompiani, 1949.

- Infanzia di Nivasio Dolcemare. Torino: Einaudi, 1973.

- Nuova Enciclopedia. Milano: Adelphi, 1977.

- Scatola Sonora. Torino: Einaudi, 1977.

Tragedia dell' infanzia. Torino: Einaudi, 1978.

A. Savinio. Catalogo della mostra di Ferrara. Ferrara: Cento, 1980.

- La nostra anima. Milano: Adelphi, 1981.

. Narrate, uomini, la vostra storia. Milano: Adelphi, 1984.

- La casa ispirata. Milano: Adelphi, 1986.

. Casa "La vita". Milano: Adelphi, 1988.

- Savinio. Catalogo. Milano: Electa, 1988. 
. Capitan Ulisse. Milano: Adelphi, 1989.

- Savinio. Catalogo. Milano: Fabbri, 1989.

Opere. Scritti dispersi tra guerra e dopo guerra (1943-1952). Ed. Leonardo

Sciascia. Milano: Bompiani, 1989.

. Achille innamorato. Milano: Adelphi, 1993.

.Hermaphrodito. Ed. Alfredo Giuliani: Milano: Adelphi, 1995. 